\title{
On the Axiomatisability of the Dual of Compact Ordered Spaces
}

\author{
Marco Abbadini ${ }^{1} \cdot$ Luca Reggio $^{2}$ (i)
}

Received: 23 September 2019 / Accepted: 30 July 2020 / Published online: 20 August 2020

(c) The Author(s) 2020

\begin{abstract}
We provide a direct and elementary proof of the fact that the category of Nachbin's compact ordered spaces is dually equivalent to an $\aleph_{1}$-ary variety of algebras. Further, we show that $\aleph_{1}$ is a sharp bound: compact ordered spaces are not dually equivalent to any SP-class of finitary algebras.
\end{abstract}

Keywords Compact ordered spaces $\cdot$ Duality $\cdot$ Axiomatisability $\cdot$ Infinitary varieties

In 1936, in his landmark paper [21], M. H. Stone described what is nowadays known as Stone duality for Boolean algebras. In modern terms, it states that the category of Boolean algebras with homomorphisms is dually equivalent to the category of totally disconnected compact Hausdorff spaces with continuous maps. If we drop the assumption of total disconnectedness, we are left with the category $\mathrm{KH}$ of compact Hausdorff spaces and continuous maps. Duskin showed in 1969 that the opposite category $\mathrm{KH}^{\mathrm{op}}$ — which, by Gelfand-Naimark duality [10], can be identified with the category of commutative unital $\mathrm{C}^{*}$-algebras-is monadic over the category of sets and functions $[8,5.15 .3]$. In fact, $\mathrm{KH}^{\text {op }}$ is equivalent to a variety of algebras. Although not finitary, this is an $\aleph_{1}$-ary variety. That is, it can be described by operations of at most countably infinite arity. A generating set of operations was exhibited by Isbell [13], while a finite axiomatisation of this variety was provided in [16]. Therefore, if we allow for infinitary operations, Stone duality for Boolean algebras can be lifted to compact Hausdorff spaces, retaining the algebraic nature.

Shortly after his paper on the duality for Boolean algebras, Stone published a generalisation of this theory to distributive lattices [23]. In his formulation, the dual category consists of the nowadays called spectral spaces and perfect maps. While spectral spaces are in general not Hausdorff, H. A. Priestley showed in 1970 that they can be equivalently described as certain compact Hausdorff spaces equipped with a partial order relation [19]. More precisely,

Communicated by M. M. Clementino.

Luca Reggio

luca.reggio@cs.ox.ac.uk

1 Dipartimento di Matematica Federigo Enriques, Università degli Studi di Milano, via Cesare Saldini 50, 20133 Milano, Italy

2 Department of Computer Science, University of Oxford, Oxford, UK 
Priestley duality states that the category of (bounded) distributive lattices is dually equivalent to the full subcategory of Nachbin's compact ordered spaces on the totally order-disconnected objects (cf. Definitions 3 and 19). As with Boolean algebras, one may ask if Priestley duality can be lifted to the category $\mathrm{KH}_{\leq}$of compact ordered spaces while retaining its algebraic nature. In [12], the authors showed that $\mathrm{KH}_{\leq}^{\mathrm{op}}$ is equivalent to an $\aleph_{1}$-ary quasi-variety and partially described its algebraic theory. In the recent work [1], the first-named author proved that $\mathrm{KH}_{\leq}^{\mathrm{op}}$ is in fact equivalent to an $\aleph_{1}$-ary variety, by providing an equational axiomatisation. The result is rather involved, and is based on an algebraic language whose finitary reduct extends the positive part of the language of MV-algebras [7].

In this note we provide a new proof of the fact that $\mathrm{KH}_{\leq}^{\mathrm{op}}$ is equivalent to an $\aleph_{1}$-ary variety, which relies only on properties of Nachbin's compact ordered spaces. The structure of our proof is the following. A well-known result in category theory, recalled in Sect. 1, characterises those categories which are equivalent to some variety of possibly infinitary algebras. A key property, which distinguishes varieties among quasi-varieties, is the effectiveness of (internal) equivalence relations. In Sect. 2 we recall some basic facts about compact ordered spaces. Further, we state Theorem 8, asserting that equivalence relations in $\mathrm{KH}_{\leq}^{\text {op }}$ are effective, and show that it implies that $\mathrm{KH}_{\leq}^{\text {op }}$ is equivalent to an $\aleph_{1}$-ary variety. Sections 3 , 4 contain the proof of Theorem 8 . First, we characterise equivalence relations on a compact ordered space $X$, in the category $\mathrm{KH}_{\leq}^{\text {op }}$, as certain pre-orders on the order-topological coproduct $X+X$. Then, we rephrase effectiveness into an order-theoretic condition and show that it is satisfied by every pre-order arising from an equivalence relation. Finally, in Sect. 5, we show that the bound $\aleph_{1}$ is best possible: $\mathrm{KH}_{\leq}$is not dually equivalent to any class of finitary algebras which is closed under taking subalgebras and Cartesian products.

Notation Given morphisms $f_{i}: X \rightarrow Y_{i}$ for $i \in\{0,1\}$, the unique morphism induced by the universal property of the product is $\left\langle f_{0}, f_{1}\right\rangle: X \rightarrow Y_{0} \times Y_{1}$. Similarly, given morphisms $g_{i}: X_{i} \rightarrow Y$ with $i \in\{0,1\}$, the coproduct map is $\left(\begin{array}{c}g_{0} \\ g_{1}\end{array}\right): X_{0}+X_{1} \rightarrow Y$. For infinite coproducts, we use the notation $\sum_{i \in I} X_{i}$. Epimorphisms are denoted by $\rightarrow$, while monomorphisms (resp. regular monomorphisms) by $\longmapsto$ (resp. $\hookrightarrow$ ). We use the symbol $\preccurlyeq$ for pre-orders, and $\leq$ for partial orders.

\section{Varieties as Categories}

In this section we provide the background needed to state a well-known characterisation of those categories which are equivalent to some (quasi-)variety of algebras. See Theorem 2 below. Throughout, all categories are assumed to be locally small and, unless otherwise stated, (quasi-)varieties admit possibly infinitary function symbols in their signatures.

Recall from [6] or [5] that a category $C$ is regular provided (i) it has finite limits, (ii) it admits coequalisers of kernel pairs, and (iii) regular epimorphisms in $C$ are stable under pullbacks. For instance, varieties and quasi-varieties of algebras (with homomorphisms) are regular categories. Those regular categories in which there is a good correspondence between regular epimorphisms and equivalence relations are called exact. In order to give a precise definition, we recall the notion of equivalence relation in a category.

Let $C$ be a category with finite limits and $A$ an object of $C$. An (internal) equivalence relation on $A$ is a subobject $\left\langle p_{0}, p_{1}\right\rangle: R \longmapsto A \times A$ satisfying the following properties:

reflexivity there exists a morphism $d: A \rightarrow R$ in $C$ such that the following diagram commutes; 


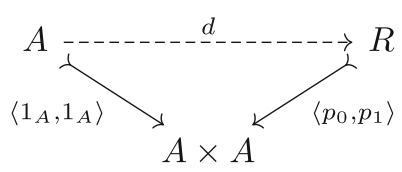

symmetry there exists a morphism $s: R \rightarrow R$ in $C$ such that the following diagram commutes;

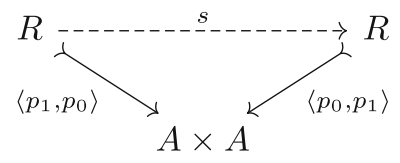

transitivity if the left-hand diagram below is a pullback square in C,
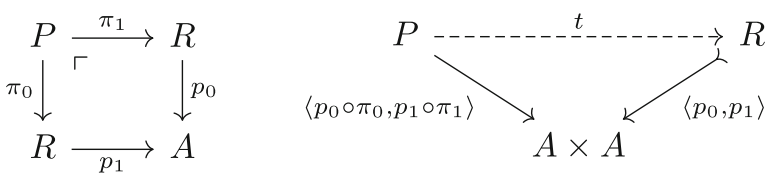

then there is a morphism $t: P \rightarrow R$ such that the right-hand diagram commutes.

Definition 1 An equivalence relation $\left\langle p_{0}, p_{1}\right\rangle: R \longmapsto A \times A$ is effective if it coincides with the kernel pair of the coequaliser of $p_{0}$ and $p_{1}$. A regular category $C$ is exact if every equivalence relation in $\mathrm{C}$ is effective.

For categories of algebras, the definition of equivalence relation given above coincides with the usual notion of congruence. Varieties of algebras are therefore exact categories, while the effective equivalence relations in quasi-varieties are the so-called relative congruences.

We need one last piece of terminology to state the desired characterisation of (quasi-) varieties of algebras. Recall that an object $G$ of a category $C$ is a regular generator if $(i)$ for every set $I$ the copower $\sum_{I} G$ exists in $C$, and (ii) for every object $A$ of $C$, the canonical morphism

$$
\sum_{\operatorname{hom}_{\mathrm{C}}(G, A)} G \rightarrow A
$$

is a regular epimorphism. Further, $G$ is regular projective if, for every morphism $f: G \rightarrow A$ and regular epimorphism $g: B \rightarrow A, f$ factors through $g$. We can now state the following well-known result.

Theorem 2 For a category C, consider the following conditions:

(1) C is regular with coequalisers of equivalence relations;

(2) Chas a regular projective regular generator $G$;

(3) every equivalence relation in $C$ is effective.

The category $C$ is equivalent to a quasi-variety if and only if it satisfies 1 and 2, and it is equivalent to a variety if and only if it satisfies 1, 2 and 3. 
The abstract characterisation of varieties and quasi-varieties has a long history in category theory, starting with the works of Lawvere, Isbell, Linton, Felscher and Duskin in the 1960s. We do not attempt here to provide an accurate historical account. For the case of quasivarieties we refer the reader to [18, Theorem 1.8], and for varieties to [6, Theorem 4.4.5] or [25]. Further, we point out that the assumption that $C$ be regular can be omitted provided C has all coequalisers, cf. [2, Theorem 3.6].

\section{Compact Ordered Spaces and Their Dual Variety}

We collect here some basic facts about compact ordered spaces, first introduced by Nachbin [17]. In particular, we describe their limits and colimits. This will come handy in the following sections.

Definition 3 A compact ordered space (or compact pospace, for short) is a pair $(X, \leq)$ where $X$ is a compact space and $\leq$ is a partial order on $X$ which is closed in the product topology of $X \times X$. We write $\mathrm{KH}_{\leq}$for the category of compact pospaces and continuous monotone maps.

A basic example of compact pospace is the unit interval $[0,1]$ equipped with the Euclidean topology and its usual total order. Note that, for any compact pospace $(X, \leq)$, the opposite order $\leq^{\mathrm{op}}=\{(x, y) \mid y \leq x\}$ is also closed in the product topology of $X \times X$. The intersection $\leq \cap \leq{ }^{\text {op }}$ coincides with the diagonal $\Delta_{X}=\{(x, x) \mid x \in X\}$, which is thus closed in $X \times X$. That is, $X$ is a Hausdorff space.

This shows that there is a forgetful functor $\mathrm{KH}_{\leq} \rightarrow \mathrm{KH}$, where $\mathrm{KH}$ denotes the category of compact Hausdorff spaces and continuous maps. On the other hand, there is also a functor $\Delta: \mathrm{KH} \rightarrow \mathrm{KH}_{\leq}$sending a compact Hausdorff space $X$ to the compact pospace $\left(X, \Delta_{X}\right)$. It is readily seen that $\Delta$ is left adjoint to the forgetful functor $\mathrm{KH}_{\leq} \rightarrow \mathrm{KH}$. In symbols,

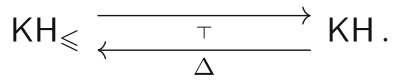

We will see in a moment that $\mathrm{KH}_{\leq}$admits all limits and colimits. By the adjunction in (1), limits in $\mathrm{KH}_{\leq}$are computed in $\mathrm{KH}$, whence in the category of sets. However, this is not the case for colimits. To circumvent this issue, we embed $\mathrm{KH}_{\leq}$in a larger category where colimits admit a simpler description.

Definition 4 A pre-ordered compact Hausdorff space is a pair $(X, \preccurlyeq)$ where $X$ is a compact Hausdorff space and $\preccurlyeq$ is a pre-order on $X$ which is closed in the product topology of $X \times X$. We write $\mathrm{KH}_{\preccurlyeq}$ for the category of pre-ordered compact Hausdorff spaces and continuous monotone maps.

Clearly, $\mathrm{KH}_{\leq}$is a full subcategory of $\mathrm{KH}_{\preccurlyeq}$ and the adjunction in (1) lifts to an adjunction between $\mathrm{KH}_{\preccurlyeq}$ and $\mathrm{KH}$. Further, the forgetful functor $\mathrm{KH}_{\preccurlyeq} \rightarrow \mathrm{KH}$ has, in addition to the left adjoint $\Delta$, also a right adjoint. Write $\nabla: \mathrm{KH} \rightarrow \mathrm{KH}_{\preccurlyeq}$ for the functor sending a compact Hausdorff space $X$ to the pre-ordered compact Hausdorff space $\left(X, \nabla_{X}\right)$, where $\nabla_{X}=X \times X$ is the improper relation on $X$. It is immediate that $\nabla$ is right adjoint to the forgetful functor $\mathrm{KH}_{\preccurlyeq} \rightarrow \mathrm{KH}$. 
Given a pre-ordered compact Hausdorff space $(X, \preccurlyeq)$, we can consider the quotient of $X$ with respect to the symmetrization of $\preccurlyeq$, that is the equivalence relation $\sim=\preccurlyeq \cap \preccurlyeq^{\mathrm{op}}$. The pre-order $\preccurlyeq$ descends to a partial order $\leq$ on the quotient space $X / \sim$, and the map

$$
\rho_{X}:(X, \preccurlyeq) \rightarrow(X / \sim, \leq)
$$

is continuous and monotone. The pair $(X / \sim, \leq)$ is readily seen to be a compact pospace. This assignment extends to a functor $\rho: \mathrm{KH}_{\preccurlyeq} \rightarrow \mathrm{KH}_{\leq}$which is left adjoint to the inclusion $\mathrm{KH}_{\leq} \rightarrow \mathrm{KH}_{\preccurlyeq}$. In other words, $\mathrm{KH}_{\leq}$is a reflective subcategory of $\mathrm{KH}_{\preccurlyeq}$.



The category $\mathrm{KH}_{\preccurlyeq}$ is complete and cocomplete because the forgetful functor $\mathrm{KH}_{\preccurlyeq} \rightarrow \mathrm{KH}$ is topological [24, Example 2], hence so is its reflective subcategory $\mathrm{KH}_{\leq}$. Since the forgetful functor $\mathrm{KH}_{\preccurlyeq} \rightarrow \mathrm{KH}$ has a right adjoint, colimits in $\mathrm{KH}_{\preccurlyeq}$ are computed in $\mathrm{KH}$. In turn, the colimit of a diagram in $\mathrm{KH}_{\leq}$can be obtained by first computing the colimit in $\mathrm{KH}_{\preccurlyeq}$, and then applying the reflector $\rho$. For more details, cf. Remark 6.

Proposition 5 The following statements hold:

(1) the regular monomorphisms in $\mathrm{KH}_{\leq}$are the continuous order-embeddings;

(2) the epimorphisms in $\mathrm{KH}_{\leq}$are the continuous monotone surjections;

(3) the unit interval $[0,1]$ is a regular injective regular cogenerator in $\mathrm{KH}_{\leq}$.

Proof See, e.g., [12, Theorem 2.6]. In particular, the unit interval is a regular cogenerator in $\mathrm{KH}_{\leq}$by [17, Chapter I, Theorems 1 and 4], and it is regular injective by [17, Chapter I, Theorem 6].

Remark 6 Let $X, Y$ be compact pospaces. Their coproduct in $\mathrm{KH}_{\preccurlyeq}$ is the disjoint union of $X$ and $Y$, with the coproduct topology and the coproduct pre-order. The latter is a compact pospace, whence it coincides with the coproduct of $X$ and $Y$ in $\mathrm{KH}_{\leq}$. Next, we describe certain pushouts in $\mathrm{KH}_{\leq}$.

Consider regular monomorphisms $f_{0}: X \hookrightarrow Y_{0}, f_{1}: X \hookrightarrow Y_{1}$ in $\mathrm{KH}_{\leq}$and their pushout in the category $\mathrm{KH}_{\preccurlyeq}$, as displayed in the following diagram.

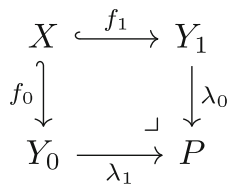

As a space, $P$ is homeomorphic to the quotient of the coproduct space $Y_{0}+Y_{1}$ with respect to the equivalence relation

$$
\left\{\left(f_{i}(x), f_{j}(x)\right) \in\left(Y_{0}+Y_{1}\right) \times\left(Y_{0}+Y_{1}\right) \mid x \in X, i, j \in\{0,1\}\right\}
$$

(transitivity follows because $f_{0}$ and $f_{1}$ are order-embeddings by item 1 in Proposition 5), which is easily seen to be closed in the product topology. Let $i \in\{0,1\}$, and write $i^{*}=1-i$. With this notation, the pre-order on $P$ is given by $\Theta=\Theta^{\prime} \cup \Theta^{\prime \prime}$, where 


$$
\begin{gathered}
\Theta^{\prime}=\left\{(p, q) \in P \times P \mid \exists i \in\{0,1\}, \exists w \in \lambda_{i}^{-1}(p), \exists w^{\prime} \in \lambda_{i}^{-1}(q), w \leq Y_{i} w^{\prime}\right\}, \\
\Theta^{\prime \prime}=\left\{(p, q) \in P \times P \mid \exists i \in\{0,1\}, \exists w \in \lambda_{i^{*}}^{-1}(p), \exists w^{\prime} \in \lambda_{i}^{-1}(q), \exists x \in X,\right. \\
\left.w \leq Y_{i} f_{i}(x) \text { and } f_{i^{*}}(x) \leq_{Y_{i^{*}}} w^{\prime}\right\} .
\end{gathered}
$$

The relation $\Theta$ is clearly reflexive, and is seen to be transitive again by item 1 in Proposition 5 . Note that $\Theta^{\prime}=\bigcup_{i \in\{0,1\}}\left(\lambda_{i} \times \lambda_{i}\right)\left(\leq_{Y_{i}}\right)$ is closed in $P \times P$. On the other hand,

$$
\begin{aligned}
\Theta^{\prime \prime} & =\bigcup_{i \in\{0,1\}}\left(\lambda_{i^{*}} \times \lambda_{i}\right)\left(\left\{\left(w, w^{\prime}\right) \in Y_{i} \times Y_{i^{*}} \mid \exists x \in X, w \leq Y_{i} f_{i}(x), f_{i^{*}}(x) \leq Y_{i^{*}} w^{\prime}\right\}\right) \\
& =\bigcup_{i \in\{0,1\}}\left(\lambda_{i^{*}} \times \lambda_{i}\right)\left(\left\{\left(w, w^{\prime}\right) \in Y_{i} \times Y_{i^{*}} \mid \exists x \in X,\left(w, w^{\prime}\right) \leq_{Y_{i} \times Y_{i^{*}}}\left(f_{i}(x), f_{i^{*}}(x)\right)\right\}\right) \\
& =\bigcup_{i \in\{0,1\}}\left(\lambda_{i^{*}} \times \lambda_{i}\right)\left(\downarrow \operatorname{Im}\left(X \stackrel{\left\langle f_{i}, f_{i^{*}}\right\rangle}{\longrightarrow} Y_{i} \times Y_{i^{*}}^{\partial}\right)\right),
\end{aligned}
$$

where $Y_{i^{*}}^{\partial}=\left(Y_{i^{*}}, \leq_{Y_{i^{*}}}^{\text {op }}\right)$. Since the downward closure $\downarrow D$ of any closed subset $D$ of a compact pospace is again closed [17, Proposition 4], we conclude that $\Theta^{\prime \prime}$ is also closed. Whence, $\Theta$ is a closed pre-order. It is not difficult to see that it is the smallest pre-order on $P$ making $\lambda_{0}$ and $\lambda_{1}$ monotone. Finally, the pushout of $f_{0}$ along $f_{1}$ in $\mathrm{KH}_{\leq}$is obtained by applying the reflector $\rho: \mathrm{KH}_{\preccurlyeq} \rightarrow \mathrm{KH}_{\leq}$to $P$.

Corollary 7 The category $\mathrm{KH}_{\leq}$is dually equivalent to a quasi-variety of algebras.

Proof By Theorem 2, it is enough to show that $(i) \mathrm{KH}_{\leq}^{\mathrm{op}}$ is regular with coequalisers of equivalence relations, and (ii) it admits a regular projective regular generator $G$.

We already observed that $\mathrm{KH}_{\leq}$is complete and cocomplete. Whence, so is $\mathrm{KH}_{\leq}^{\text {op }}$. To show that $\mathrm{KH}_{\leq}^{\mathrm{op}}$ is regular, it suffices to prove that regular monos, i.e. continuous order-embeddings, are stable under pushouts in $\mathrm{KH}_{\leq}$. Pushouts in $\mathrm{KH}_{\leq}$can be computed by first taking the pushout in $\mathrm{KH}_{\preccurlyeq}$, and then composing with the reflection map. Reasoning as in Remark 6, it is not difficult to see that the pushout of a continuous order-embedding in $\mathrm{KH}_{\preccurlyeq}$ is again a continuous order-embedding. Further, composing with the reflection yields again a continuous orderembedding, i.e. a regular mono in $\mathrm{KH}_{\leq}$. This proves $(i)$. In turn, (ii) follows at once from item 3 in Proposition 5, by setting $G=[0,1]$. We mention that one may also deduce ( $i$ ) from (ii) and the fact that $\mathrm{KH}_{\leq}^{\mathrm{op}}$ is cocomplete, cf. [2, Theorem 3.6].

The latter fact was already observed in [12] where, in addition, the authors provide a description of an $\aleph_{1}$-ary quasi-variety dually equivalent to $\mathrm{KH}_{\leq}$(see Theorem 3.15 in op. cit.). Our main contribution consists in a direct proof of the following result:

Theorem 8 Every equivalence relation in $\mathrm{KH}_{\leq}^{\mathrm{op}}$ is effective.

A proof of the previous theorem is provided in Sects. 3, 4. We conclude this section by observing that Theorem 8 implies that $\mathrm{KH}_{\leq}^{\mathrm{op}}$ is equivalent to an $\aleph_{1}$-ary variety of algebras, that is a variety of algebras in a language consisting of function symbols of at most countably infinite arity.

Corollary 9 The category $\mathrm{KH}_{\leq}$is dually equivalent to an $\aleph_{1}$-ary variety of algebras.

Proof By Corollary 7, we know that $\mathrm{KH}_{\leq}$is dually equivalent to a quasi-variety of algebras. Theorems 2 and 8 entail that $\mathrm{KH}_{\leq}$is in fact dually equivalent to a variety of algebras. Indeed, 
$\mathrm{KH}_{\leq}^{\mathrm{op}}$ is equivalent to the category of Eilenberg-Moore algebras for the monad induced by the adjunction

$$
\sum_{-}[0,1] \dashv \operatorname{hom}_{\mathrm{KH}} \mathrm{op}([0,1],-): \mathrm{KH}_{\leq}^{\mathrm{op}} \rightarrow \text { Set } .
$$

The latter is equivalent to an $\aleph_{1}$-ary variety of algebras if, and only if, the monad preserves $\aleph_{1}$ directed colimits. It suffices to show that, for every set $I$ and continuous monotone function $f:[0,1]^{I} \rightarrow[0,1]$, there is a countable subset $J \subseteq I$ such that $f$ factors through the projection $[0,1]^{I} \rightarrow[0,1]^{J}$. In turn, this is a consequence of the Stone-Weierstrass Theorem for compact Hausdorff spaces [22].

\section{Equivalence Co-relations on Compact Ordered Spaces}

In this section we provide a description of equivalence relations in the category $\mathrm{KH}_{\leq}^{\mathrm{op}}$, which will then be exploited in the next section to prove that equivalence relations in $\mathrm{KH}_{\leq}^{\mathrm{op}}$ are effective.

To start with, we dualise the notion of subobject. Given a compact pospace $X$, a quotient object of $X$ is a subobject of $X$ in the category $\mathrm{KH}_{\leq}^{\mathrm{op}}$. The poset of quotient objects of $X$ is denoted by $\mathbf{Q}(X)$. Explicitly, $\mathbf{Q}(X)$ is the poset of (equivalence classes of) epimorphisms with domain $X$, where $f_{1}: X \rightarrow Y_{1}$ is below $f_{2}: X \rightarrow Y_{2}$ whenever there exists $g: Y_{2} \rightarrow Y_{1}$ such that $g \circ f_{2}=f_{1}$.

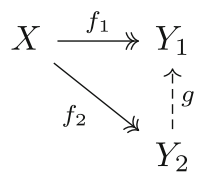

Remark 10 We warn the reader that our terminology is non-standard. By a quotient object we do not mean a regular epimorphism, but what may be called a co-subobject (not every epimorphism in $\mathrm{KH}_{\leq}$is regular).

By definition, an equivalence relation on $X$ in the opposite category $\mathrm{KH}_{\leq}^{\mathrm{op}}$ is a subobject of $X \times X$ (where the product is computed in $\mathrm{KH}_{\leq}^{\mathrm{op}}$ ) which is reflexive, symmetric and transitive. This corresponds to a quotient object $\left(\begin{array}{l}q_{0} \\ q_{1}\end{array}\right): X+X \rightarrow S$ of the compact pospace $X+X$ satisfying the dual properties:

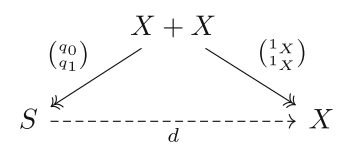

co-reflexivity

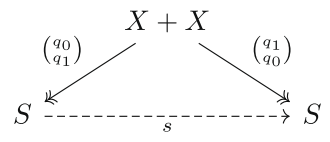

co-symmetry

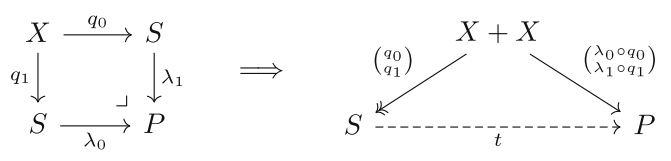

co-transitivity 
A quotient object of $X+X$ which satisfies the three properties above will be called an equivalence co-relation on $X$. The key observation is that equivalence co-relations are more manageable than their duals, because quotient objects of $X$ are in bijection with certain pre-orders on $X$.

Indeed, if $f:\left(X, \leq_{X}\right) \rightarrow\left(Y, \leq_{Y}\right)$ is an epimorphism in $\mathrm{KH}_{\leq}$, then

$$
\preccurlyeq f=\left\{\left(x_{1}, x_{2}\right) \in X \times X \mid f\left(x_{1}\right) \leq_{Y} f\left(x_{2}\right)\right\}
$$

is a pre-order on $X$. The monotonicity of $f$ entails $\leq_{X} \subseteq \preccurlyeq f$. Further, recalling that epimorphisms in $\mathrm{KH}_{\leq}$are precisely the continuous monotone surjections (see item 2 in Proposition 5), we see that $\preccurlyeq f$ is closed in $X \times X$ because it coincides with the preimage of $\leq_{Y}$ under the continuous map $f \times f: X \times X \rightarrow Y \times Y$. Let us denote by $\mathbf{P}(X)$ the poset of all closed pre-orders on $X$ which extend $\leq X$, ordered by reverse inclusion. By the previous discussion, there is a map $\mathbf{Q}(X) \rightarrow \mathbf{P}(X)$ sending $f$ to $\preccurlyeq f$. This function is well-defined, as $\preccurlyeq f$ does not depend on the choice of a representative in the equivalence class of $f$. Conversely, given a pre-order $\preccurlyeq$ in $\mathbf{P}(X)$, consider its symmetrization $\sim=\preccurlyeq \cap \preccurlyeq$ op . The space $X / \sim$, equipped with the quotient topology, is compact. The direct image of $\preccurlyeq$ under the quotient map is a partial order on $X / \sim$, and it is closed because so is $\preccurlyeq$. Moreover, since $\leq_{X} \subseteq \preccurlyeq$, we get an epimorphism

$$
X \rightarrow X / \sim
$$

in $\mathrm{KH}_{\leq}$. Taking its equivalence class, we obtain an element of $\mathbf{Q}(X)$. The following fact follows easily.

Lemma 11 For every compact pospace $X$, the assignments

$$
(f: X \rightarrow Y) \mapsto \preccurlyeq f \text { and } \preccurlyeq \mapsto(X \rightarrow X / \sim)
$$

induce an isomorphism between the posets $\boldsymbol{P}(X)$ and $\boldsymbol{Q}(X)$.

Remark 12 Assume $f_{1}: X \rightarrow Y_{1}$ and $f_{2}: X \rightarrow Y_{2}$ are surjective morphisms in $\mathrm{KH}_{\leq}$. By Lemma 11, there exists $g: Y_{1} \rightarrow Y_{2}$ such that $g \circ f_{1}=f_{2}$ if, and only if, $\forall x, y \in X$, $f_{1}(x) \leq f_{1}(y)$ implies $f_{2}(x) \leq f_{2}(y)$. In fact, it is not difficult to see that this is true even if $f_{2}$ is not surjective, as we can factor it as a surjective map followed by an injective one.

Recall from Remark 6 that the compact pospace $X+X$ is isomorphic to the disjoint union

$$
\{(x, 0) \mid x \in X\} \cup\{(x, 1) \mid x \in X\},
$$

equipped with the coproduct topology and the coproduct order.

Notation We denote the elements of $X+X$ by $(x, i),(y, j), \ldots$ where $i, j$ vary in $\{0,1\}$. Further, $i^{*}$ stands for $1-i$. For example, $\left(x, 1^{*}\right)=(x, 0)$.

For the rest of this section, we fix a quotient object $\left(\begin{array}{c}q_{0} \\ q_{1}\end{array}\right): X+X \rightarrow S$ of a compact pospace $X$. We write $\preccurlyeq\left(q_{q_{1}}^{q_{0}}\right)$, or simply $\preccurlyeq s$, for the associated pre-order on $X+X$. We say that $\preccurlyeq s$ is co-reflexive (co-symmetric, co-transitive) if so is $\left(\begin{array}{c}q_{0} \\ q_{1}\end{array}\right)$. To improve readability, we write $[(x, i)]$ instead of $\left(\begin{array}{l}q_{0} \\ q_{1}\end{array}\right)(x, i)$.

Lemma 13 The following statements hold:

(1) the pre-order $\preccurlyeq s$ is co-reflexive if, and only if, $(x, i) \preccurlyeq s(y, j)$ entails $x \leq y$;

(2) the pre-order $\preccurlyeq s$ is co-symmetric if, and only if, $(x, i) \preccurlyeq s \quad(y, j)$ entails $\left(x, i^{*}\right) \preccurlyeq s$ $\left(y, j^{*}\right)$. 
Proof (1) By definition, $\preccurlyeq s$ is co-reflexive if, and only if, $\left(\begin{array}{l}q_{0} \\ q_{1}\end{array}\right): X+X \rightarrow S$ is above $\left(\begin{array}{l}1_{X} \\ 1_{X}\end{array}\right): X+X \rightarrow X$ in the poset $\mathbf{Q}(X+X)$. By Lemma 11, this is equivalent to $\preccurlyeq S \subseteq \preccurlyeq\left(\begin{array}{l}1_{X} \\ 1_{X}\end{array}\right)$. Given $(x, i),(y, j) \in X+X$, we have

$$
(x, i) \preccurlyeq\left(\begin{array}{c}
1_{X}^{X} \\
1_{X}
\end{array}\right)(y, j) \Longleftrightarrow x \leq y .
$$

It follows that the pre-order $\preccurlyeq s$ is co-reflexive if, and only if, $(x, i) \preccurlyeq s \quad(y, j)$ entails $x \leq y$.

(2) Again, by definition, $\preccurlyeq s$ is co-symmetric if and only if $\left(\begin{array}{l}q_{0} \\ q_{1}\end{array}\right): X+X \rightarrow S$ is above $\left(\begin{array}{l}q_{1} \\ q_{0}\end{array}\right): X+X \rightarrow S$ in $\mathbf{Q}(X+X)$. By Lemma 11, this happens exactly when $\preccurlyeq s \subseteq \preccurlyeq\left(\begin{array}{l}q_{1} \\ q_{0}\end{array}\right)$. Given $(x, i),(y, j) \in X+X$,

$$
(x, i) \preccurlyeq\left(\begin{array}{l}
q_{1} \\
q_{0}
\end{array}\right)(y, j) \Longleftrightarrow\left(x, i^{*}\right) \preccurlyeq S\left(y, j^{*}\right) .
$$

Therefore, the pre-order $\preccurlyeq s$ is co-symmetric if, and only if, $(x, i) \preccurlyeq s \quad(y, j)$ entails $\left(x, i^{*}\right) \preccurlyeq s\left(y, j^{*}\right)$.

Lemma 14 Assume the pre-order $\preccurlyeq s$ is co-reflexive. Then it is co-transitive if, and only if,

$$
(x, i) \preccurlyeq s\left(y, i^{*}\right) \Longrightarrow \exists z \in X\left[(x, i) \preccurlyeq S\left(z, i^{*}\right) \text { and }(z, i) \preccurlyeq S\left(y, i^{*}\right)\right] .
$$

Proof Recall that $\preccurlyeq S$ is co-transitive if, and only if, given a pushout square in $\mathrm{KH}_{\leq}$as in the left-hand diagram below,
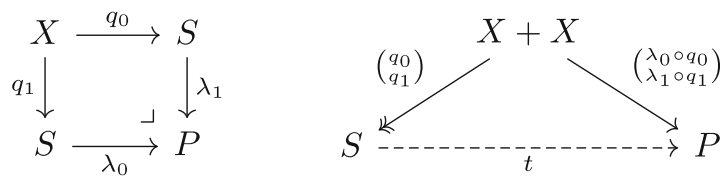

there is $t: S \rightarrow P$ making the right-hand diagram commute. By Remark 12, such a $t$ exists precisely when, for every $(x, i),(y, j) \in X+X,(x, i) \preccurlyeq s \quad(y, j)$ implies $\left(\begin{array}{l}\lambda_{0} \circ q_{0} \\ \lambda_{1} \circ q_{1}\end{array}\right)(x, i) \leq$ $\left(\begin{array}{l}\lambda_{0} \circ q_{0} \\ \lambda_{1} \circ q_{1}\end{array}\right)(y, j)$, i.e. $\lambda_{i}([(x, i)]) \leq \lambda_{j}([(y, j)])$. Recall that $\preccurlyeq s$ is co-reflexive provided $q_{0}$ and $q_{1}$ are both sections of a morphism $d: S \rightarrow X$. In particular, $q_{0}$ and $q_{1}$ are regular monomorphisms in $\mathrm{KH}_{\leq}$. Thus, by Remark $6, \lambda_{i}([(x, i)]) \leq \lambda_{j}([(y, j)])$ if, and only if,

$[i=j$ and $(x, i) \preccurlyeq s(y, j)]$ or $[i \neq j$ and $\exists z \in X$ s.t. $(x, i) \preccurlyeq s(z, j)$ and $(z, i) \preccurlyeq s(y, j)]$.

We conclude that $\preccurlyeq s$ is co-reflexive if, and only if, equation (2) holds whenever $(x, i) \preccurlyeq s$ $(y, j)$. In turn, this is equivalent to the condition in the statement of the lemma.

From Lemmas 13 and 14, we obtain the following characterisation of equivalence corelations in $\mathrm{KH}_{\leq}$.

Proposition 15 The pre-order $\preccurlyeq s$ is an equivalence co-relation on $X$ if, and only if,

$$
(x, i) \preccurlyeq s(y, j) \Longrightarrow\left[x \leq y \text { and }\left(x, i^{*}\right) \preccurlyeq s\left(y, j^{*}\right)\right]
$$

and

$$
(x, i) \preccurlyeq s\left(y, i^{*}\right) \Longrightarrow \exists z \in X\left[(x, i) \preccurlyeq s\left(z, i^{*}\right) \text { and }(z, i) \preccurlyeq s\left(y, i^{*}\right)\right] \text {. }
$$




\section{Proof of Theorem 8}

Assume $\left(\begin{array}{c}q_{0} \\ q_{1}\end{array}\right): X+X \rightarrow S$ is an equivalence co-relation on $X$. Dualising Definition 1, we say that $\left(\begin{array}{l}q_{0} \\ q_{1}\end{array}\right)$ is effective provided it coincides with the co-kernel pair of its equaliser. That is, provided the following is a pushout square in $\mathrm{KH}_{\leq}$,

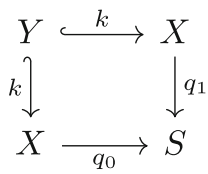

where $k$ is the equaliser of $q_{0}, q_{1}: X \rightrightarrows S$ in $\mathrm{KH}_{\leq}$. Also, we say that the pre-order $\preccurlyeq s$ is effective if so is the corresponding quotient object. By item 1 in Proposition 5, the space $Y$ can be identified with a closed subset of $X$, equipped with the induced order and topology. Define the relation $\preccurlyeq^{Y}$ on $X+X$ as follows:

$$
(x, i) \preccurlyeq^{Y}(y, j) \Longleftrightarrow(i=j \text { and } x \leq y) \text { or }\left(i^{*}=j \text { and } \exists z \in Y \text { s.t. } x \leq z \leq y\right) \text {. }
$$

Lemma $16 \preccurlyeq^{Y}$ is the pre-order on $X+X$ associated with the pushout of the inclusion $Y \hookrightarrow X$ along itself.

Proof This is an immediate consequence of Remark 6.

For the next proposition, recall that $\sim_{S}=\preccurlyeq S \cap \preccurlyeq_{S}^{\text {op }}$ is the symmetrization of the pre-order $\preccurlyeq s$.

Proposition 17 The equivalence co-relation $\preccurlyeq s$ is effective if, and only if,

$$
(x, i) \preccurlyeq S\left(y, i^{*}\right) \Longrightarrow \exists z \in X\left[x \leq z \leq y \text { and }(z, i) \sim_{S}\left(z, i^{*}\right)\right] .
$$

Proof Recall that the equivalence co-relation $\preccurlyeq s$ is effective if, and only if, the diagram in (3) is a pushout in $\mathrm{KH}_{\leq}$. In turn, by Lemma 16, this is equivalent to saying that $\preccurlyeq s=\preccurlyeq{ }^{Y}$. Since $Y=\left\{x \in X \mid(x, \bar{i}) \sim_{S}\left(x, i^{*}\right)\right\}$,

$$
(x, i) \preccurlyeq^{Y}(y, j) \Longleftrightarrow \exists z \in X\left[x \leq z \leq y \text { and }(z, i) \sim_{S}\left(z, i^{*}\right)\right] .
$$

Therefore, to settle the statement, it suffices to show that the inclusion $\preccurlyeq^{Y} \subseteq \preccurlyeq s$ is always satisfied.

Note that any equivalence co-relation $\preccurlyeq$ on $X$ satisfies $(x, i) \preccurlyeq(y, i)$ if, and only if, $x \leq y$. The left-to-right implication follows from item 1 in Lemma 13, while the right-to-left implication holds because $\preccurlyeq$ extends the coproduct order of $X+X$. Whence, $(x, i) \preccurlyeq^{Y}(y, i)$ if, and only if, $(x, i) \preccurlyeq s \quad(y, i)$. Suppose now $(x, i) \preccurlyeq^{Y}\left(y, i^{*}\right)$, and let $z \in Y$ satisfy $x \leq z \leq y$. We have

$$
(x, i) \preccurlyeq s(z, i) \sim_{S}\left(z, i^{*}\right) \preccurlyeq s\left(y, i^{*}\right),
$$

where the two inequalities hold because $\preccurlyeq s$ extends the partial order of $X+X$. Therefore, $\preccurlyeq^{Y} \subseteq \preccurlyeq s$.

We can finally prove Theorem 8, stating that every equivalence relation in $\mathrm{KH}_{\leq}^{\text {op }}$ is effective. 
Proof of Theorem 8 Let $(X, \leq)$ be a compact pospace and $\preccurlyeq$ an equivalence co-relation on $X$. In view of Proposition 17 it is enough to show that, whenever $(x, i) \preccurlyeq\left(y, i^{*}\right)$, there is $z \in X$ such that

$$
x \leq z \leq y \text { and }(z, i) \sim\left(z, i^{*}\right) .
$$

Fix arbitrary $x, y \in X$ and $i \in\{0,1\}$ satisfying $(x, i) \preccurlyeq\left(y, i^{*}\right)$, and set

$$
\Omega=\left\{u \in X \mid(x, i) \preccurlyeq\left(u, i^{*}\right) \text { and }(u, i) \preccurlyeq\left(y, i^{*}\right)\right\} .
$$

The idea is to apply Zorn's Lemma to show that $\Omega$ has a maximal element $z$ satisfying the desired properties.

First, note that $\Omega$ is non-empty because $\preccurlyeq$ is co-transitive, cf. Lemma 14 . We claim that every non-empty chain $C \subseteq \Omega$ admits an upper bound in $\Omega$.

Every directed set in a compact pospace has a supremum, which coincides with the topological limit of the set regarded as a net [11, Proposition VI.1.3]. Thus, $C$ has a supremum $s$ in $X$, which belongs to the topological closure $\bar{C}$ of $C$.

Claim $\Omega$ is a closed subset of $X$.

Proof The set $\Omega$ can be written as the intersection of the sets

$$
\Omega_{1}=\left\{u \in X \mid(x, i) \preccurlyeq\left(u, i^{*}\right)\right\} \text { and } \Omega_{2}=\left\{u \in X \mid(u, i) \preccurlyeq\left(y, i^{*}\right)\right\} .
$$

Hence, it is enough to show that $\Omega_{1}$ and $\Omega_{2}$ are closed in $X$. We show that $\Omega_{1}$ is closed. The proof for $\Omega_{2}$ is the same, mutatis mutandis. The set $\Omega_{1}$ is the preimage, under the coproduct injection $\iota_{i}: X \rightarrow X+X$, of

$$
\uparrow(x, i)=\{(w, j) \in X+X \mid(x, i) \preccurlyeq(w, j)\} .
$$

Since $\preccurlyeq$ is a closed pre-order on $X+X$, the set $\uparrow(x, i)$ is closed in $X+X$ [17, Proposition 1]. Therefore, its preimage $\Omega_{1}$ is closed in $X$.

The previous claim entails that $s \in \bar{C} \subseteq \Omega$, i.e. $C$ has a supremum in $\Omega$. Hence, every non-empty chain in $\Omega$ admits an upper bound. By Zorn's Lemma, $\Omega$ has a maximal element $z$. By co-reflexivity of $\preccurlyeq$ (see item 1 in Lemma 13$),(x, i) \preccurlyeq\left(z, i^{*}\right)$ and $(z, i) \preccurlyeq\left(y, i^{*}\right)$ imply $x \leq z \leq y$. It remains to show that $(z, i) \sim\left(z, i^{*}\right)$.

Since $(z, i) \preccurlyeq\left(y, i^{*}\right)$, by co-transitivity of $\preccurlyeq($ cf. Lemma 14), there is $u \in X$ such that $(z, i) \preccurlyeq\left(u, i^{*}\right)$ and $(u, i) \preccurlyeq\left(y, i^{*}\right)$. Also, $(x, i) \preccurlyeq(z, i)$ because $\preccurlyeq$ extends the partial order of $X$. Thus $(x, i) \preccurlyeq(z, i) \preccurlyeq\left(u, i^{*}\right)$, which implies $u \in \Omega$. By co-reflexivity, $(z, i) \preccurlyeq\left(u, i^{*}\right)$ entails $z \leq u$. Since $z$ is maximal, it must be $z=u$. Therefore, $(z, i) \preccurlyeq\left(z, i^{*}\right)$. By cosymmetry (see item 2 in Lemma 13), we conclude that $(z, i) \sim\left(z, i^{*}\right)$.

We saw that, for every compact pospace $X$ and closed subset $Y \subseteq X$, there is a pre-order $\preccurlyeq^{Y}$ on $X+X$ given as in (4). In fact, by Lemma $16, \preccurlyeq^{Y}$ is the equivalence co-relation on $X$ associated with the pushout of the inclusion $Y \hookrightarrow X$ along itself. Conversely, every equivalence co-relation $\preccurlyeq$ on $X$ yields a closed subset of $X$, namely

$$
\Phi(\preccurlyeq)=\left\{x \in X \mid(x, i) \sim\left(x, i^{*}\right)\right\} .
$$

Corollary 18 For every compact pospace $X$, the assignments

$$
\preccurlyeq \mapsto \Phi(\preccurlyeq) \text { and }(Y \hookrightarrow X) \mapsto \preccurlyeq^{Y}
$$

yield an isomorphism between the poset of equivalence co-relations on $X$ and the poset of closed subsets of $X$. 
Proof The two maps are clearly monotone. For any closed subset $Y \subseteq X$, we have $\Phi\left(\preccurlyeq^{Y}\right)=Y$ because

$$
(x, i) \sim^{Y}\left(x, i^{*}\right) \Longleftrightarrow \exists z \in Y \text { s.t. } x \leq z \leq x \Longleftrightarrow x \in Y .
$$

Moreover, it follows at once from Theorem 8 and Proposition 17 that, for any equivalence co-relation $\preccurlyeq$ on $X, \preccurlyeq \subseteq \preccurlyeq{ }^{\Phi(\preccurlyeq)}$. For the converse inclusion, see the proof of Proposition 17 .

\section{Epilogue: Negative Axiomatisability Results}

In the previous sections, we have given a direct proof of the fact that the category $\mathrm{KH}_{\leq}$ of compact ordered spaces is dually equivalent to an $\aleph_{1}$-ary variety of algebras. One may wonder whether it is necessary to resort to infinitary operations. In this section we show that $\mathrm{KH}_{\leq}^{\mathrm{op}}$ is not equivalent to any SP-class of finitary algebras (i.e., one closed under subalgebras and Cartesian products), let alone a finitary (quasi-)variety.

Recall that an object $A$ of a category $C$ is (Gabriel-Ulmer) finitely presentable if the covariant hom-functor $\operatorname{hom}_{\mathrm{C}}(A,-): \mathrm{C} \rightarrow$ Set preserves directed colimits. See [9, Definition 6.1] or [3, Definition 1.1]. Further, $\mathrm{C}$ is finitely accessible provided it has directed colimits and there exists a set $S$ of its objects such that ( $i$ ) each object of $S$ is finitely presentable, and (ii) each object of $C$ is a directed colimit of objects in $S$. See [3, Definition 2.1].

For example, finitary varieties and finitary quasi-varieties (with homomorphisms) are finitely accessible categories, cf. [3, Corollary 3.7 and Theorem 3.24]. Recall the following definition:

Definition 19 A Priestley space is a compact pospace $(X, \leq)$ which is totally orderdisconnected, i.e. for all $x, y$ with $x \not \leq y$ there is a clopen $C \subseteq X$ which is an up-set for $\leq$ and satisfies $x \in C$ but $y \notin C$.

Lemma 20 A compact pospace is a Priestley space if, and only if, it is the codirected limit in $\mathrm{KH}_{\leq}$of finite posets equipped with the discrete topologies.

Proof This result is folklore. For a proof see, e.g., [14, Corollary VI.3.3].

Denote by PSp the full subcategory of $\mathrm{KH}_{\leq}$defined by all Priestley spaces. By a result of Priestley [19], $\mathrm{PSp}^{\text {op }}$ is equivalent to the category of bounded distributive lattices with homomorphisms. In particular, $\mathrm{PSp}^{\mathrm{op}}$ is a finitely accessible category. The following result is an adaptation of [16, Proposition 1.2] to the ordered case.

Theorem 21 Let $F$ be a full subcategory of $\mathrm{KH}_{\leq}$extending PSp. If $\mathrm{F}^{\mathrm{op}}$ is a finitely accessible category - in particular, if $F^{\mathrm{op}}$ is a finitary variety or a finitary quasi-variety - then $F=P S p$.

Proof It suffices to show that every object in F is a Priestley space. We claim that every finitely copresentable object in F (i.e. one which is finitely presentable when regarded as an object of $\left.\mathrm{F}^{\mathrm{op}}\right)$ is finite.

Let $(X, \leq)$ be an arbitrary finitely copresentable object in F. Consider an epimorphism $\gamma: Y \rightarrow X$ in $\mathrm{KH}_{\leq}$with $Y$ a Priestley space. (E.g., let $Y=\beta|X|$ be the Čech-Stone compactification of the underlying set of $X$ equipped with the discrete topology, and $\gamma:(\beta|X|,=) \rightarrow$ $(X, \leq)$ the unique continuous extension of the identity function $|X| \rightarrow|X|)$. By Lemma 20, $Y$ is the codirected limit in $\mathrm{KH}_{\leq}$of finite posets $\left\{Y_{i}\right\}_{i \in I}$ with the discrete topologies. Denote 
by $\alpha_{i}: Y \rightarrow Y_{i}$ the $i$-th limit arrow. Since $Y$ lies in $\mathrm{F}$, and the full embedding $\mathrm{F} \rightarrow \mathrm{KH}_{\leq}$ reflects limits, $Y$ is in fact the codirected limit of $\left\{Y_{i}\right\}_{i \in I}$ in $\mathrm{F}$.

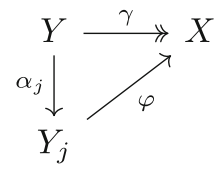

The object $X$ being finitely copresentable in $\mathrm{F}$, there are $j \in I$ and a morphism $\varphi: Y_{j} \rightarrow X$ such that $\gamma=\varphi \circ \alpha_{j}$. The map $\gamma$ is surjective, hence so is $\varphi$. This shows that $X$ is finite, and thus the claim is settled.

Since $F^{o p}$ is finitely accessible, every object of $F$ is the codirected limit of finitely copresentable objects. Using again the fact that the full embedding $\mathrm{F} \rightarrow \mathrm{KH}_{\leq}$reflects limits, we deduce from Lemma 20 that every object of $F$ is a Priestley space, as was to be shown. Finally, we have already observed that finitary varieties and finitary quasi-varieties are finitely accessible categories.

Corollary $22 K H_{\leq}^{\mathrm{op}}$ is not equivalent to any SP-class of finitary algebras.

Proof By Theorem 8, every equivalence relation in $\mathrm{KH}_{\leq}^{\mathrm{op}}$ is effective. In turn, Banaschewski observed in [4] that every SP-class of finitary algebras in which every equivalence relation is effective is a variety of algebras. The statement then follows from Theorem 21.

Remark 23 In a recent work, Lieberman, Rosický and Vasey [15] proved that the opposite of the category $\mathrm{KH}$ of compact Hausdorff spaces is not equivalent to any elementary class of structures, with morphisms all the homomorphisms. In fact, they show that there exists no faithful functor $\mathrm{KH}^{\mathrm{op}} \rightarrow$ Set which preserves directed colimits. Since directed colimits in elementary classes are concrete [20], the preceding statement follows. This implies that $\mathrm{KH}_{\leq}^{\mathrm{op}}$ is not equivalent to any elementary class of structures. Indeed, note that the embedding $\Delta: \mathrm{KH}^{\mathrm{op}} \rightarrow \mathrm{KH}_{\leq}^{\mathrm{op}}$ (cf. equation (1)) preserves directed colimits. Hence, if there were a faithful functor $F: \mathrm{KH}_{\leq}^{\mathrm{op}} \rightarrow$ Set preserving directed colimits, the composition $F \circ \Delta: \mathrm{KH}^{\mathrm{op}} \rightarrow$ Set would also be a faithful functor preserving directed colimits, contradicting the aforementioned result. This shows that $\mathrm{KH}_{\leq}^{\mathrm{op}}$ cannot be equivalent to an elementary class of structures with morphisms all the homomorphisms.

Acknowledgements The first-named author expresses his gratitude to J. Somaglia for some helpful discussions on convergence of nets. The second-named author is grateful to the Institute of Computer Science of the Czech Academy of Sciences and the Mathematical Institute of the University of Bern, where this work was carried out, for providing a highly stimulating environment. Finally, we are most grateful to the anonymous referee for their comments, which helped us to improve the presentation of our results.

Open Access This article is licensed under a Creative Commons Attribution 4.0 International License, which permits use, sharing, adaptation, distribution and reproduction in any medium or format, as long as you give appropriate credit to the original author(s) and the source, provide a link to the Creative Commons licence, and indicate if changes were made. The images or other third party material in this article are included in the article's Creative Commons licence, unless indicated otherwise in a credit line to the material. If material is not included in the article's Creative Commons licence and your intended use is not permitted by statutory regulation or exceeds the permitted use, you will need to obtain permission directly from the copyright holder. To view a copy of this licence, visit http://creativecommons.org/licenses/by/4.0/. 


\section{References}

1. Abbadini, M.: The dual of compact ordered spaces is a variety. Theory Appl. Categ. 34, 1401-1439 (2019)

2. Adámek, J.: On quasivarieties and varieties as categories. Stud. Log. 78(1-2), 7-33 (2004)

3. Adámek, J., Rosický, J.: Locally Presentable and Accessible Categories. London Mathematical Society Lecture Note Series, vol. 189. Cambridge University Press, Cambridge (1994)

4. Banaschewski, B.: On categories of algebras equivalent to a variety. Algebra Univ. 16(2), 264-267 (1983)

5. Barr, M., Grillet, P.A., van Osdol, D.: Exact Categories and Categories of Sheaves. Lecture Notes in Mathematics, vol. 236. Springer, Berlin (1971)

6. Borceux, F.: Handbook of Categorical Algebra 2. Encyclopedia of Mathematics and its Applications, vol. 51. Cambridge University Press, Cambridge (1994)

7. Cignoli, R.L.O., D’Ottaviano, I.M.L., Mundici, D.: Algebraic Foundations of Many-Valued Reasoning. Trends in Logic-Studia Logica Library, vol. 7. Kluwer Academic Publishers, Dordrecht (2000)

8. Duskin, J.: Variations on Beck's tripleability criterion. In: Mac Lane, S. (ed.) Reports of the Midwest Category Seminar, III, pp. 74-129. Springer, Berlin (1969)

9. Gabriel, P., Ulmer, F.: Lokal präsentierbare Kategorien. Lecture Notes in Mathematics, vol. 221. Springer, Berlin (1971)

10. Gelfand, I., Neumark, M.: On the imbedding of normed rings into the ring of operators in Hilbert space. Rec. Math. [Mat. Sbornik] N.S. 12(54), 197-213 (1943)

11. Gierz, G., Hofmann, K.H., Keimel, K., Lawson, J.D., Mislove, M.W., Scott, D.S.: A Compendium of Continuous Lattices. Springer, Berlin (1980)

12. Hofmann, D., Neves, R., Nora, P.: Generating the algebraic theory of $C(X)$ : the case of partially ordered compact spaces. Theory Appl. Categ. 33, 276-295 (2018)

13. Isbell, J.: Generating the algebraic theory of $C(X)$. Algebra Univ. 15(2), 153-155 (1982)

14. Johnstone, P.T.: Stone Spaces. Cambridge Studies in Advanced Mathematics, vol. 3. Cambridge University Press, Cambridge (1986)

15. Lieberman, M., Rosický, J., Vasey, S.: Hilbert spaces and $\mathrm{C}^{*}$-algebras are not finitely concrete. arXiv: 1908.10200

16. Marra, V., Reggio, L.: Stone duality above dimension zero: axiomatising the algebraic theory of $\mathrm{C}(X)$. Adv. Math. 307, 253-287 (2017)

17. Nachbin, L.: Topology and order. Translated from the Portuguese by Lulu Bechtolsheim. Van Nostrand Mathematical Studies, No. 4. D. Van Nostrand Co., Inc., Princeton, N.J.-Toronto, Ont.-London (1965)

18. Pedicchio, M.C.: On $k$-permutability for categories of $T$-algebras. In: Logic and algebra (Pontignano, 1994), volume 180 of Lecture Notes in Pure and Appl. Math., pp 637-646. Dekker, New York (1996)

19. Priestley, H.A.: Representation of distributive lattices by means of ordered Stone spaces. Bull. Lond. Math. Soc. 2, 186-190 (1970)

20. Richter, M.: Limites in Kategorien von Relationalsystemen. Z. Math. Logik Grundlagen Math. 17, 75-90 (1971)

21. Stone, M.H.: The theory of representations for Boolean algebras. Trans. Am. Math. Soc. 40(1), 37-111 (1936)

22. Stone, M.H.: Applications of the theory of Boolean rings to general topology. Trans. Am. Math. Soc. 41(3), 375-481 (1937)

23. Stone, M.H.: Topological representations of distributive lattices and brouwerian logics. Časopis pro pěsiování matematiky a fysiky 67, 1-25 (1938)

24. Tholen, W.: Ordered topological structures. Topol. Appl. 156(12), 2148-2157 (2009)

25. Vitale, E.M.: On the characterization of monadic categories over SET. Cahiers Topologie Géom. Différentielle Catég. 35(4), 351-358 (1994)

Publisher's Note Springer Nature remains neutral with regard to jurisdictional claims in published maps and institutional affiliations. 